\title{
EDITORIAL
}

\section{HEALTH AND WELL-BEING OF STUDENTS AT HIGHER EDUCATION INSTITUTIONS - TIME FOR URGENT ACTION?}

Welcome to this special themed edition of the Central European Journal of Public Health. The seven articles published in this issue, dedicated to the health and well-being of higher education institutions students, present research pertaining to students from several high, low and middle-income countries across several continents. Taken together, CEJPH hopes that these studies bestow a rich and multi-perspective offering on the subject. Indeed, geographically, these students are enrolled at universities, colleges, and higher technical institutions located in culturally and politically diverse nations such as Egypt, the United Kingdom, Palestine, the Netherlands, Libya, and South Africa. Academically and demographically, the presented research assessed male and female students at different study years, a range of faculties, and across many academic disciplines. Methodologically, the studies employed quantitative methods (using generous sample sizes) or qualitative methods (using strategic sampling). Conceptually, the presented studies collectively report a wide range of socio-demographic characteristics, lifestyle features and other variables that influence and impact on students' health and well-being. These include body image, smoking and smoking-associated health risks, nutrition, climate change, physical activity (PA), psychosomatic symptoms and health complaints, ethical concerns, and mental health challenges such as stress and depressive symptoms. Unfortunately, the findings of these studies raise concerns, as they seem to converge into one direction: that it is time for serious action if educators, health authorities and policy practitioners are to focus their much needed attention in order to maintain and improve the health and well-being of higher education students. Many examples within this issue serve to illustrate this urgent need for action.

As for some of the factors that pertain directly to student health, in Egypt, high proportions of the 3,271 students that were sampled felt fatigue, difficulties to concentrate, headache, mood swings, nervousness/anxiety, and sleep disorder symptoms sometimes/very often in the last 12 months. In the Gaza Strip, Palestine, slightly more than half of the 1,104 participants reported ever smoking, students' knowledge of existing anti-smoking legislation in Palestine was low, and almost all students reported that there were no smoking cessation centres in Palestine, or did not know whether such centres existed. In the United Kingdom, across 3,706 students from Northern Ireland, England and Wales, consuming 'unhealthy' foods (e.g. sweets, cookies, snacks, fast food) was significantly and positively associated with perceived stress (females only) and depressive symptoms (both genders), suggesting that interventions aimed at reducing depressive symptoms and stress among students could also result in the consumption of healthier foods and/or vice versa. In Libya, of the 1,300 students surveyed, about $44 \%$ achieved the international guidelines/recommendations for muscle strengthening PA, while only $11 \%$ achieved the international guidelines for vigorous PA, $29 \%$ for moderate to vigorous PA, $21 \%$ for walking, 4.8\% for moderate PA (excluding walking), and female students were particularly at risk for low PA. As for body image concerns (BIC), again in the United Kingdom, more females (35\%) than males ( $8 \%$ ) had moderate/marked concerns with their body image, and among females, there was a co-occurrence of depressive symptoms and BIC, suggesting that more attention is required to the gender-specific correlates of BIC for tailoring evidence based interventions for females and for males.

In terms of the factors that pertain more broadly to the general well-being of students (e.g. climate change), in the Netherlands, driven by feelings of guilt and responsibility, an ecological worldview and desire to play a positive role in society, university students voiced concerns about the increasing social and economic inequalities between the global North and South, and the transfer of materialistic value systems and unsustainable practices from developed to developing countries. In South Africa, as recipients of information on climate change, students' well-being was negatively affected by the media's pessimism of communicating risks and the inadequate/restricted networking of communicating solutions and strategies. Similarly, as contributors to information on climate change, students faced barriers to their efficacy and agency that included socio-cultural inequalities (e.g. race, language), and a lack of formal platforms for community recognition, policy consultation and collaboration.

Despite that the educated youth represent the main foundation of the future of our nations, the global picture of student health and well-being is not encouraging. Yet little efforts are invested, with initiatives that seem to be reactive rather than proactive, mainly curative rather than preventive, dispersed rather than systematic, or simply 'too little-too late'. For instance, whilst some countries monitor their university students' satisfaction in terms of their educational experience, much fewer countries systematically monitor the health/well-being of their students (student health barometer). Such a continuously evolving knowledge base would be essential for the strategic planning and informed tailoring of interventions. In addition, universities will need to do more in order to be truly 'Health Promoting Universities'. Anecdotal evidence suggests that in some countries, there are few PA opportunities on campus (particularly gender-specific), and few campus health clinics manned with qualified personnel. Likewise, in other countries, on campus legislation (e.g. smoking ban) seems not appropriately enforced. These are some mere examples, but the list is long. The WHO calls for stronger focus on adolescent health, and the Health 2020 policy framework has 'investing in health through a life-course approach and empowering people' as one of four priority areas for policy action. It is certainly time for more systematic, coordinated, evidence based, multi-level, intersectoral actions driven by stakeholder partnerships comprising college students, educators and campus managers, funding agencies and granting bodies, academic researchers and evaluators, and health planners and other authorities, aimed at the health and well-being of our university student populations. After all, these young adults are every nation's most valuable assets. 